\title{
Application of Base Force Element Method to Mesomechanics Analysis for Recycled Aggregate Concrete
}

\author{
Yijiang Peng, ${ }^{1}$ Yinghua Liu, ${ }^{2}$ Jiwei Pu, ${ }^{1}$ and Lijuan Zhang' \\ ${ }^{1}$ Key Lab of Urban Security \& Disaster Engineering, Department of Civil Engineering, Beijing University of Technology, \\ Beijing 100124, China \\ ${ }^{2}$ AML, Department of Engineering Mechanics, Tsinghua University, Beijing 100084, China
}

Correspondence should be addressed to Yinghua Liu; yhliu@mail.tsinghua.edu.cn

Received 18 July 2013; Accepted 23 August 2013

Academic Editor: Song Cen

Copyright (C) 2013 Yijiang Peng et al. This is an open access article distributed under the Creative Commons Attribution License, which permits unrestricted use, distribution, and reproduction in any medium, provided the original work is properly cited.

\begin{abstract}
The base force element method (BFEM) on potential energy principle is used to analyze recycled aggregate concrete (RAC) on mesolevel. The model of BFEM with triangular element is derived. The recycled aggregate concrete is taken as five-phase composites consisting of natural coarse aggregate, new mortar, new interfacial transition zone (ITZ), old mortar, and old ITZ on meso-level. The random aggregate model is used to simulate the mesostructure of recycled aggregate concrete. The mechanics properties of uniaxial compression and tension tests for RAC are simulated using the BFEM, respectively. The simulation results agree with the test results. This research method is a new way for investigating fracture mechanism and numerical simulation of mechanics properties for recycled aggregate concrete.
\end{abstract}

\section{Introduction}

"Concrete" is considered as heterogeneous composites whose mechanical performance is much related to the microstructure of material. The mechanical performances are usually obtained using experimental methods. However, testing usually consumes a large amount of manpower and material resources, and test results are usually more discrete. In order to overcome this defect, the concept of numerical concrete was presented by Wittmann et al. [1] based on micromechanics. Subsequently, some scholars did some creative works in this field and made a number of models. Among them, the two important models are the lattice model and the random aggregate model. For example, Schlangen et al. [2,3] applied the lattice model to simulate the failure mechanism of concrete. Liu and Wang [4] adopted the random aggregate model to simulate cracking process of concrete using FEM. Peng et al. [5] adopted the random aggregate model to simulate the mechanics properties of rolled compacted concrete on meso-level using FEM. Du et al. [6] simulated the failure mechanism of beam under impact loading and triangular cyclic loading by using displacement-controlled FEM, stressstrain curves, and dynamic bending strengths of specimens.
"Recycled aggregate concrete" which is used as a green building material has attracted more and more researchers with the shortage of resources and an increasing number of construction wastes. They have carried out a series of experiments and some conclusions have been obtained. An overview of study on recycled aggregate concrete has been given by Xiao et al. [7]. However, because of the complexity of recycled coarse aggregates, conclusions made by different researchers are usually not very accordant, even opposite sometimes. To remove effects of experimental conditions, some numerical researches on meso-level were considered. For example, numerical simulation on stress-strain curve of recycled aggregate concrete was made by Xiao et al. [8] with lattice model under uniaxial compression. A method on mesomechanics analysis was proposed by Peng et al. [9, 16] and Zhou et al. [10] using FEM for recycled aggregate concrete based on random aggregate model. However, the numerical researches on the damage mechanism for recycled aggregate concrete material have just begun.

In recent years, a new type of finite element method, the base force element method (BFEM), has been developed by Peng et al. [9, 11-17] based on the concept of the base forces 


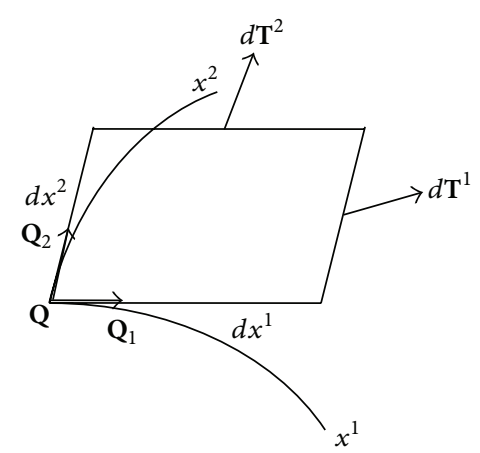

FIgURE 1: The base forces.

by Gao [18]. In this paper, the BFEM on potential energy principle is used to analyze recycled aggregate concrete (RAC) on meso-level. The mechanics properties of recycled aggregate concrete in uniaxial compression and tension tests are simulated using the BFEM, respectively. The simulation results agree with the test results. This research method is a new way for investigating fracture mechanism and numerical simulation of mechanics properties of recycled aggregate concrete.

\section{Basic Equation}

Consider a two-dimensional domain of solid medium; let $\mathbf{x}^{\alpha}(\alpha=1,2)$ denote the Lagrangian coordinate system, where $\mathbf{P}$ and $\mathbf{Q}$ are the position vectors of a material point before and after deformation, respectively. Two triads for original and current configurations can be defined as follows:

$$
\mathbf{P}_{\alpha}=\frac{\partial \mathbf{P}}{\partial x^{\alpha}}, \quad \mathbf{Q}_{\alpha}=\frac{\partial \mathbf{Q}}{\partial x^{\alpha}} .
$$

Let $\mathbf{u}$ denote the displacement of a point; then

$$
\mathbf{u}=\mathbf{Q}-\mathbf{P} .
$$

The gradient of displacement $\mathbf{u}_{\alpha}$ can be written as follows:

$$
\mathbf{u}_{\alpha}=\frac{\partial \mathbf{u}}{\partial x^{\alpha}}=\mathbf{Q}_{\alpha}-\mathbf{P}_{\alpha} .
$$

Then, the Green strain $\varepsilon$ can be written as

$$
\varepsilon=\frac{1}{2}\left(\mathbf{u}_{i} \otimes \mathbf{P}^{i}+\mathbf{P}^{i} \otimes \mathbf{u}_{i}\right) .
$$

In order to describe the stress state at a point $\mathbf{Q}$, a parallelogram with the edges $\mathrm{d} x^{1} \mathbf{Q}_{1}, \mathrm{~d} x^{2} \mathbf{Q}_{2}$ is shown in Figure 1. Define

$$
\mathbf{T}^{\alpha}=\frac{\mathrm{d} T^{\alpha}}{\mathrm{d} x^{\alpha+1}}, \quad \mathrm{~d} x^{\alpha} \longrightarrow 0
$$

where $3=1$ for indexes. Quantities $\mathbf{T}^{\alpha}(\alpha=1,2)$ are called the base forces at point $\mathbf{Q}$ in the two-dimensional coordinate system $x^{\alpha}$.

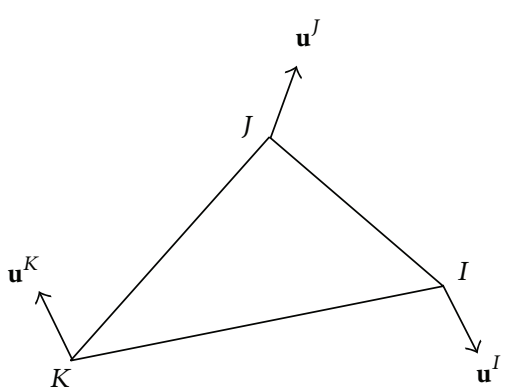

FIGURE 2: A triangular element.

According to the definitions of various stress tensors, the relation between the base forces and various stress tensors can be given. The Cauchy stress is

$$
\boldsymbol{\sigma}=\frac{1}{A_{\mathrm{Q}}} \mathbf{T}^{\alpha} \otimes \mathbf{Q}_{\alpha} .
$$

Further, the base forces are given as follows:

$$
\mathbf{T}^{\alpha}=\rho A_{Q} \frac{\partial W}{\partial \mathbf{u}_{\alpha}}=\rho_{0} A_{P} \frac{\partial W}{\partial \mathbf{u}_{\alpha}},
$$

in which $W$ is the strain energy density and $\rho_{0}$ is the mass density before deformation.

Equation (7) expresses the $\mathrm{T}^{\alpha}$ by strain energy directly. Thus, $\mathbf{u}_{\alpha}$ is just the conjugate variable of $\mathbf{T}^{\alpha}$. It can be seen that the mechanics problem can be completely established by means of $\mathbf{T}^{\alpha}$ and $\mathbf{u}_{\alpha}$.

\section{Model of BFEM with Triangular Element}

We will derive explicit expressions for stiffness matrices of a triangular element now, based on the concept of "base forces." Consider a triangular element with boundary $S$ as shown in Figure 2.

For the small displacement case, the real strain $\varepsilon$ can be replaced by $\overline{\boldsymbol{\varepsilon}}$. We can obtain the average strain in element as

$$
\bar{\varepsilon}=\frac{1}{A} \int_{A} \varepsilon \mathrm{d} A
$$

in which $A$ is the area of element.

Substituting (4) into (8), we have

$$
\overline{\boldsymbol{\varepsilon}}=\frac{1}{2 A} \int_{A}\left(\mathbf{u}_{\alpha} \otimes \mathbf{P}^{\alpha}+\mathbf{P}^{\alpha} \otimes \mathbf{u}_{\alpha}\right) \mathrm{d} A .
$$

Using Green's theorem, (9) becomes

$$
\overline{\boldsymbol{\varepsilon}}=\frac{1}{2 A} \int_{S}(\mathbf{u} \otimes \mathbf{n}+\mathbf{n} \otimes \mathbf{u}) \mathrm{d} s,
$$

where $\mathbf{n}$ is the current normal of boundary $S$.

When the element is small enough, (10) can be written as

$$
\overline{\boldsymbol{\varepsilon}}=\frac{1}{2 A} \sum_{i=1}^{3} L_{i}\left(\mathbf{u}_{i} \otimes \mathbf{n}_{i}+\mathbf{n}_{i} \otimes \mathbf{u}_{i}\right),
$$


where $L_{i}$ is the length of edge $i(i=1,2,3), \mathbf{n}_{i}$ denotes the external normal of edge $i(i=1,2,3)$, and $\mathbf{u}_{i}$ is the displacement of geometric center of edge $i(i=1,2,3)$.

Further, we assume that any edge of the triangular element in the deformation process keeps its edges straight lines. Then, we can obtain the following expression for $\mathbf{u}_{i}$ :

$$
\mathbf{u}_{i}=\frac{1}{2}\left(\mathbf{u}_{I}+\mathbf{u}_{J}\right),
$$

where $\mathbf{u}_{I}$ and $\mathbf{u}_{J}$ denote the displacements of both ends of edge $i(i=1,2,3)$, respectively.

Substituting (12) into (11) yields

$$
\overline{\boldsymbol{\varepsilon}}=\frac{1}{2 A}\left(\mathbf{u}_{I} \otimes \mathbf{m}^{I}+\mathbf{m}^{I} \otimes \mathbf{u}_{I}\right) .
$$
$\mathbf{m}^{I}$ is

The summation rule is implied in the above equation, and

$$
\mathbf{m}^{I}=\frac{1}{2}\left(L_{I J} \mathbf{n}^{I J}+L_{I K} \mathbf{n}^{I K}\right),
$$

where $L_{I J}$ and $L_{I K}$ are the lengths of edges $I J$ and $I K$ and $\mathbf{n}^{I J}$ and $\mathbf{n}^{I K}$ denote the external normals of edges $I J$ and $I K$, respectively.

Then, for an isotropic material, the strain energy in the element is reduced to

$$
W_{D}=\frac{A E}{2(1+\nu)}\left[\frac{\nu}{1-2 \nu}(\overline{\boldsymbol{\varepsilon}}: \mathbf{U})^{2}+\overline{\boldsymbol{\varepsilon}}: \overline{\boldsymbol{\varepsilon}}\right],
$$

in which $E$ is Young's modulus and $v$ is Poisson's ratio.

Substituting (13) into (15) we have

$$
\begin{gathered}
W_{D}=\frac{E}{4 A(1+\nu)}\left[\frac{2 \nu}{1-2 \nu}\left(\mathbf{u}_{I} \cdot \mathbf{m}^{I}\right)^{2}+\left(\mathbf{u}_{I} \cdot \mathbf{u}_{J}\right) m^{I J}\right. \\
\left.+\left(\mathbf{u}_{I} \cdot \mathbf{m}^{I}\right)\left(\mathbf{u}_{J} \cdot \mathbf{m}^{I}\right)\right],
\end{gathered}
$$

where

$$
m^{I J}=\mathbf{m}^{I} \cdot \mathbf{m}^{J} .
$$

From (16), we can obtain the force acting on this element at node $I$

$$
\mathbf{f}^{I}=\frac{\partial W_{D}}{\partial \mathbf{u}^{I}}=\mathbf{K}^{I J} \cdot \mathbf{u}_{J}
$$

where

$$
\mathbf{K}^{I J}=\frac{E}{2 A(1+v)}\left[\frac{2 v}{1-2 v} \mathbf{m}^{I} \otimes \mathbf{m}^{J}+m^{I J} \mathbf{U}+\mathbf{m}^{J} \otimes \mathbf{m}^{I}\right] .
$$

Here, $\mathbf{K}^{I J}$ is a second-order tensor that is called the stiffness matrix.

The characteristics of the stiffness matrix $\mathbf{K}^{I J}$ compared with the traditional FEM are as follows. (1) This expression of stiffness matrix $\mathbf{K}^{I J}$ can easily be extended to apply to arbitrary polygonal elements problem in two dimensions or arbitrary polyhedral element problem in three dimensions.

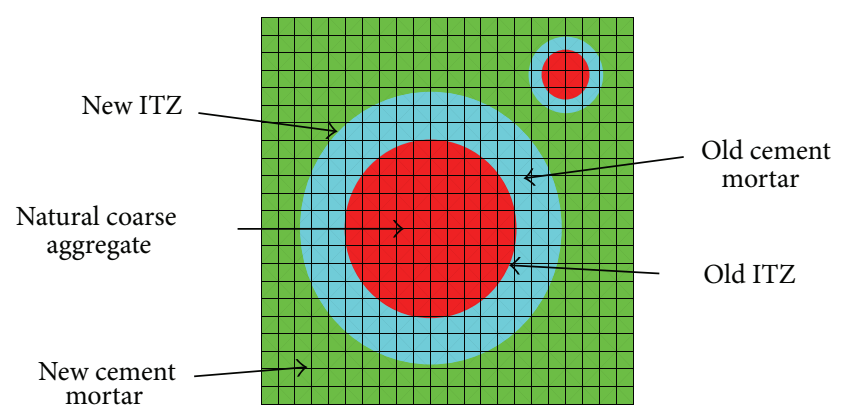

Figure 3: Attribute recognition figure.

(2) The expression of the stiffness matrix $\mathbf{K}^{I J}$ is a precise expression, and it is not necessary to introduce Gauss's integral for calculating the stiffness coefficient at a point. (3) This expression of $\mathbf{K}^{I J}$ can be used for calculating the stiffness of various elements with a unified method. (4) This expression of stiffness matrix $\mathbf{K}^{I J}$ can be used in any coordinate system. (5) The method of constructing the stiffness matrix does not regulate the introduction of interpolation.

The model of the base force element method will be used to analyze the damage problem for recycled aggregate concrete and to analyze the relationships between mesostructure and macroscopic mechanical performance of recycled aggregate concrete.

\section{Random Aggregate Model for RAC}

Based on the Fuller grading curve, Walraven and Reinhardt [19] put the three-dimensional grading curve into the probability of any point located in the sectional plane of specimens, and its expression is as follows:

$$
\begin{aligned}
P_{c}\left(D<D_{0}\right) & \\
=P_{k}( & 1.065\left(\frac{D_{0}}{D_{\max }}\right)^{1 / 2}-0.053\left(\frac{D_{0}}{D_{\max }}\right)^{4} \\
& -0.012\left(\frac{D_{0}}{D_{\max }}\right)^{6}-0.0045\left(\frac{D_{0}}{D_{\max }}\right)^{8} \\
& \left.-0.0025\left(\frac{D_{0}}{D_{\max }}\right)^{10}\right),
\end{aligned}
$$

where $P_{k}$ is the volume percentage of aggregate volume among the specimens, in general $P_{k}=0.75, D_{0}$ is the diameter of sieve pore, and $D_{\max }$ is the maximum aggregate size.

According to (20), the numbers of coarse aggregate particles with various sizes can be obtained. By Monte Carlo method, random to create the centroid coordinates of all kinds of coarse aggregate particles, namely, to generate random aggregate model as Figure 3. According to the projection method, we dissect the specimens of RAC with different phases of materials. Then, the phase of recycled coarse aggregate, the phase of new hardened cement, the phase of old hardened cement, and the phase of new and old interfacial transition zone (ITZ) can be judged by a computer code. 
TABLE 1: Material parameters of numerical simulations.

\begin{tabular}{|c|c|c|c|c|c|c|}
\hline Materials & Elastic modulus/GPa & Poisson's ratio & Tensile strength/MPa & $\lambda$ & $\eta$ & $\xi$ \\
\hline Natural coarse aggregate & 70 & 0.16 & 10 & 0.1 & 5 & 10 \\
\hline Old ITZ & 13 & 0.2 & 2 & 0.1 & 3 & 10 \\
\hline Old cement mortar & 25 & 0.22 & 2.5 & 0.1 & 4 & 10 \\
\hline New ITZ & 15 & 0.2 & 2 & 0.1 & 3 & 10 \\
\hline New cement mortar & 30 & 0.22 & 3 & 0.1 & 4 & 10 \\
\hline
\end{tabular}

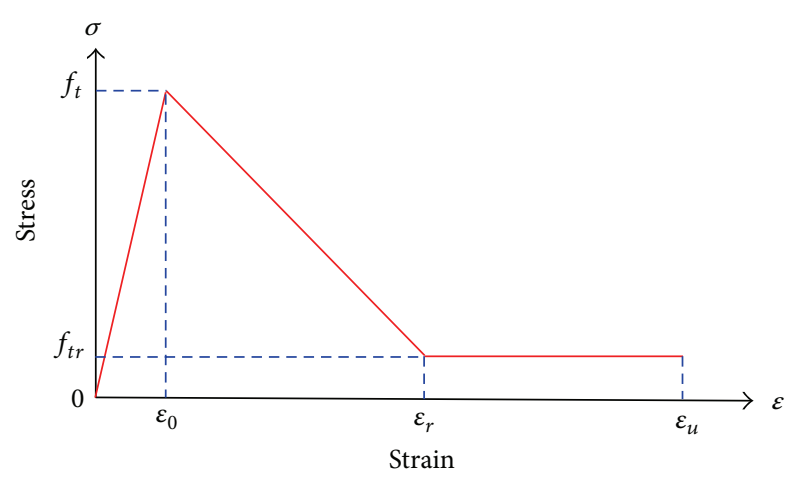

FIgURE 4: Bilinear damage model.

\section{Damage Model of Materials}

Components of RAC such as recycled coarse aggregate, new mortar, old mortar, new interfacial transition zone (new ITZ), and old interfacial transition zone (old ITZ) are basically quasi-brittle material, whose failure patterns are mainly brittle failure. In this paper, according to the characteristics of recycled aggregate concrete on meso-structure, the damage degradation of recycled aggregate concrete is described by the bilinear damage model, and the failure principal is the criterion of maximum tensile strain. Damage constitutive model is defined as $\widetilde{E}=E(1-D)$ as shown in Figure 4, where the damage factor $D$ can be expressed as follows:

$$
D= \begin{cases}0 & \varepsilon<\varepsilon_{0} \\ 1-\frac{\eta-\lambda}{\eta-1} \frac{\varepsilon_{0}}{\varepsilon}+\frac{1-\lambda}{\eta-1} & \varepsilon_{0}<\varepsilon \leq \varepsilon_{r} \\ 1-\lambda \frac{\varepsilon_{0}}{\varepsilon} & \varepsilon_{r}<\varepsilon \leq \varepsilon_{u} \\ 1 & \varepsilon>\varepsilon_{u}\end{cases}
$$

where $f_{t}$ is the tensile strength of material, the residual tensile strength is defined as $f_{t r}=\lambda f_{t}$, the residual strength coefficient $\lambda$ ranges from 0 to 1 , the residual strain is $\varepsilon_{r}=$ $\eta \varepsilon_{0}, \eta$ is the residual strain coefficient, the ultimate strain is defined as $\varepsilon_{u}=\xi \varepsilon_{0}$, where $\xi$ is ultimate strain coefficient, and $\varepsilon$ is principal tensile strain of element.

\section{Numerical Examples}

According to the test results achieved from the experiments, material parameters of recycled aggregate concrete are

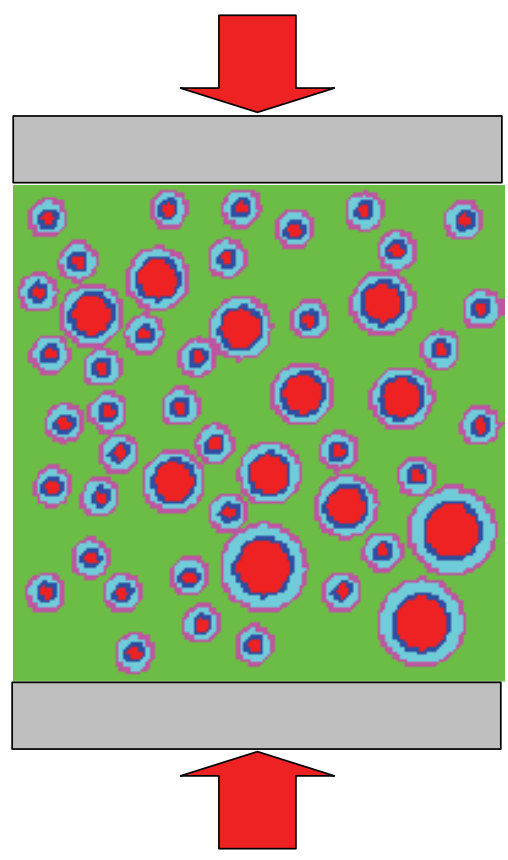

FIGURE 5: Numerical analysis of uniaxial compressive strength for RAC specimen.

selected for the numerical simulations. Material parameters of numerical simulations are shown in Table 1.

6.1. Simulation on Uniaxial Compressive Strength of RAC. Based on the uniaxial compression experiments, uniaxial compressive strengths for RAC specimens are investigated using the BFEM as shown in Figure 5.

The dimension of compression specimen is $100 \mathrm{~mm} \times$ $100 \mathrm{~mm} \times 100 \mathrm{~mm}$. The numbers of large aggregates (representative size is $17.5 \mathrm{~mm}$ ), middle aggregates (representative size is $12.5 \mathrm{~mm}$ ), and small aggregates (representative size is $7.5 \mathrm{~mm}$ ) are 3,9 , and 37 , respectively. In order to simplify the numerical calculation, the three-dimensional structure is schematized as a plane stress problem and the geometry of the analytical specimen is $100 \mathrm{~mm} \times 100 \mathrm{~mm}$. The random aggregate models of a RAC specimen are shown in Figure 6. Three specimens with different random aggregate distribution are simulated and the mean compressive strength of these specimens is obtained.

The uniaxial compressive stress-strain curve of recycled aggregate concrete is as shown in Figure 7. 


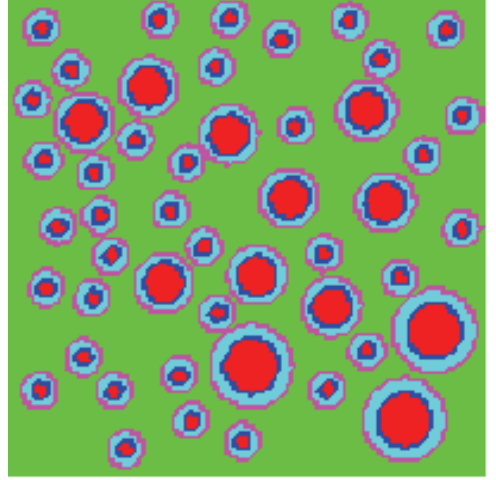

Specimen 1

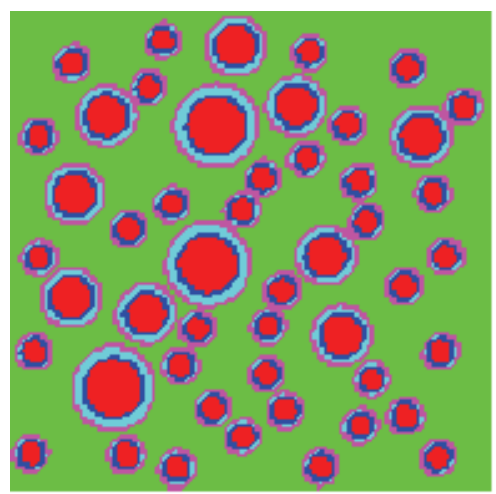

Specimen 2

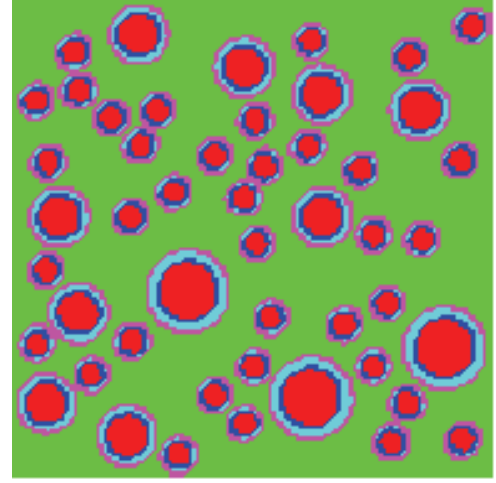

Specimen 3

FIGURE 6: Random aggregate models of recycled aggregate concrete with a gradation.

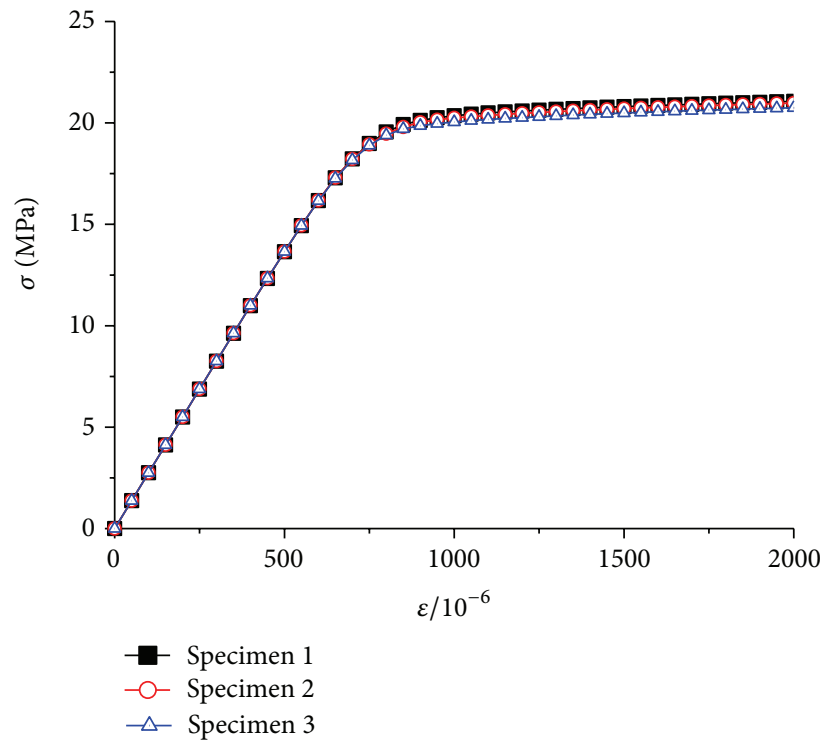

FIGURE 7: Compressive stress-strain curve of recycled aggregate concrete.

The compressive strengths of the three specimens are 20.36 MPa, 20.25 MPa, and 20.05 MPa. The average uniaxial compressive strength of the specimen group is $20.22 \mathrm{MPa}$. The result of BFEM on mesodamage analysis for RAC is consistent with the test results [20].

The propagation process of cracks of the RAC specimen by uniaxial compression is shown in Figure 8 .

6.2. Simulation on Uniaxial Tensile Strength of RAC. Based on the uniaxial tension experiments, uniaxial tensile strengths for RAC specimens are investigated using the BFEM as shown in Figure 9.

The dimension of tension specimen is $100 \mathrm{~mm} \times 100 \mathrm{~mm}$ $\times 100 \mathrm{~mm}$. The numbers of large aggregates (representative size is $17.5 \mathrm{~mm}$ ), middle aggregates (representative size is $12.5 \mathrm{~mm}$ ), and small aggregates (representative size is $7.5 \mathrm{~mm}$ ) are 3,9 , and 37 , respectively. In order to simplify the numerical calculation, the three-dimensional structure is schematized as a plane stress problem and the geometry of the analytical specimen is $100 \mathrm{~mm} \times 100 \mathrm{~mm}$. The random aggregate model of a RAC specimen is shown in Figure 6. Three specimens with different random aggregate distribution are simulated and the mean tensile strength of these specimens is obtained.

The uniaxial tensile stress-strain curve of recycled aggregate concrete is as shown in Figure 10.

The tensile strengths of the three specimens are $2.64 \mathrm{MPa}$, $2.44 \mathrm{MPa}$, and 2.63 MPa. The average uniaxial tensile strength of the specimen group is $2.57 \mathrm{MPa}$. The result of BFEM on meso-damage analysis for RAC is consistent with the test results [21].

The propagation process of cracks of the RAC specimen by uniaxial tension is shown in Figure 11.

\section{Conclusions}

(1) In this paper, a model of the base force element method (BFEM) is proposed for the damage analysis problem and is used to simulate the relations of mesostructure and macro-strength of recycled aggregate concrete (RAC). The characteristics of the BFEM are that the expression of the stiffness matrix $\mathbf{K}^{I J}$ is a precise expression and it is not necessary to introduce the interpolation function and Gauss's integral for calculating the stiffness coefficient at a point. The numerical results show that this method can be used for damage analysis of the RAC.

(2) In order to simulate the meso-structure of recycled aggregate concrete material, recycled aggregate concrete is taken as five-phase composites consisting of natural coarse aggregate, new mortar, new interfacial transition zone (ITZ), old mortar, and old ITZ on meso-level in this paper. The random aggregate models are used for the numerical simulations of uniaxial compressive and tensile performances of recycled aggregate concrete, respectively. The results of the BFEM on meso-damage analysis for RAC are consistent with the test results. 

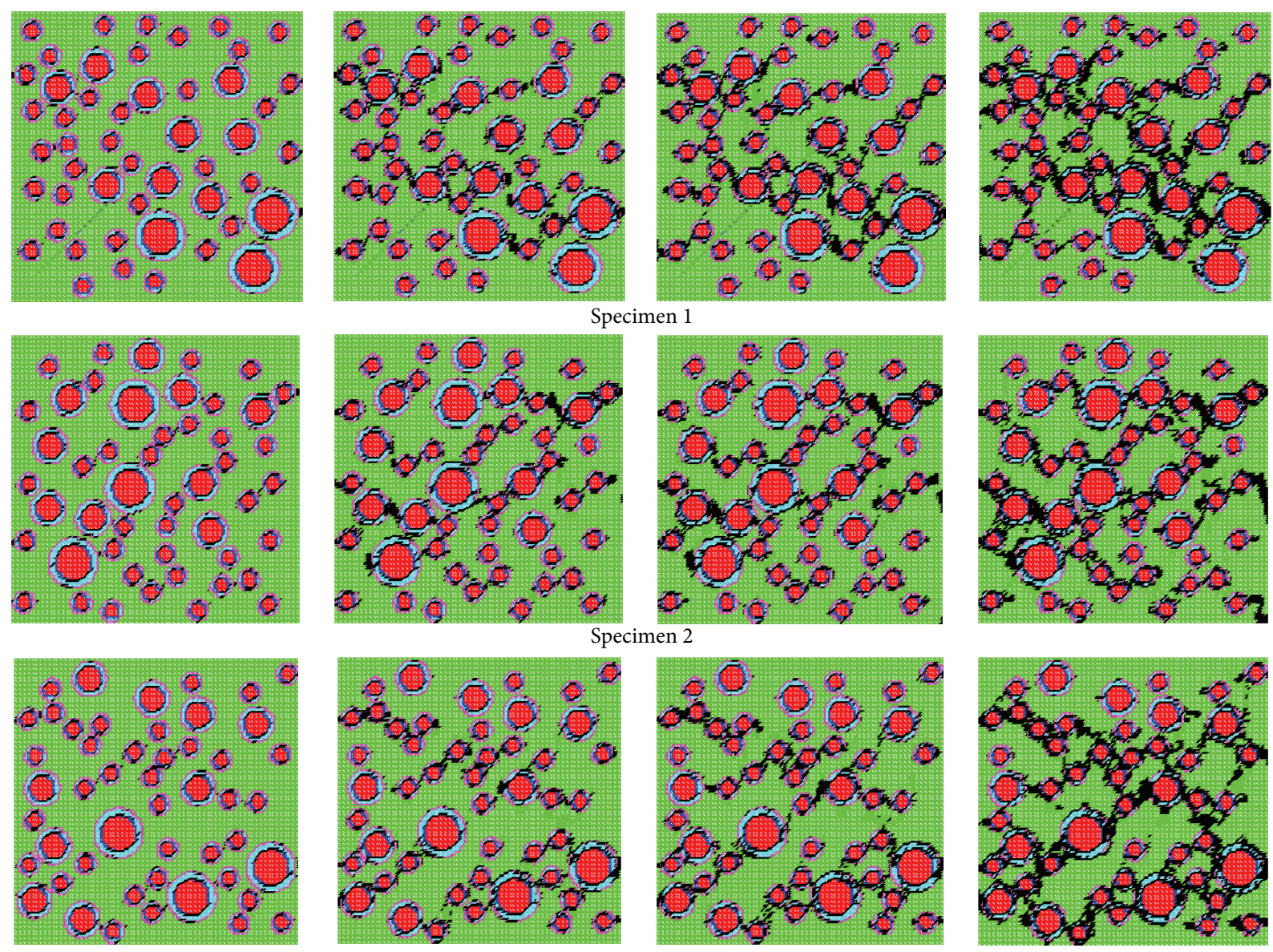

FIGURE 8: Propagation process of cracks of the RAC specimen by uniaxial compression.

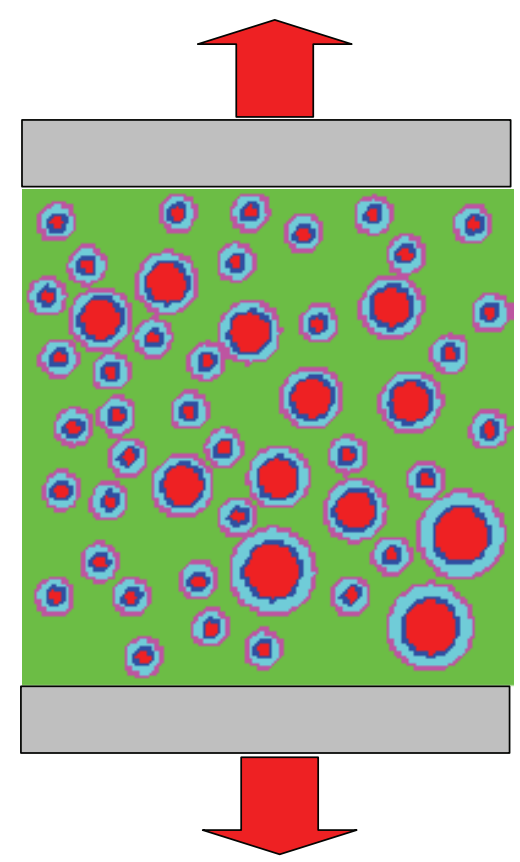

FIgURE 9: Numerical analysis of uniaxial tensile strength for RAC specimen.

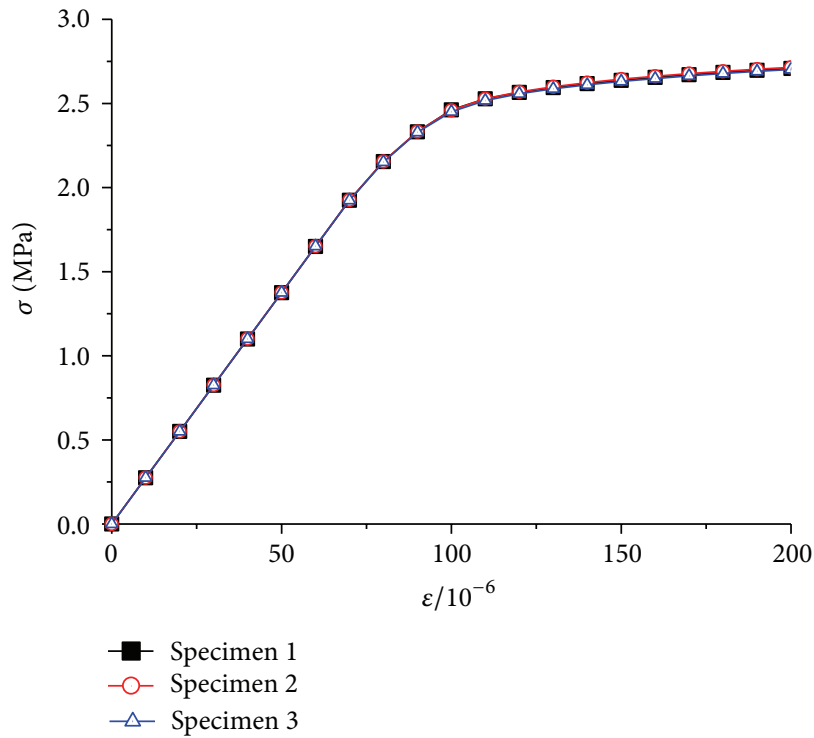

FIGURE 10: Tensile stress-strain curve of recycled aggregate concrete.

(3) The numerical simulation provides a new way for research on mechanical properties of recycled aggregate concrete. 

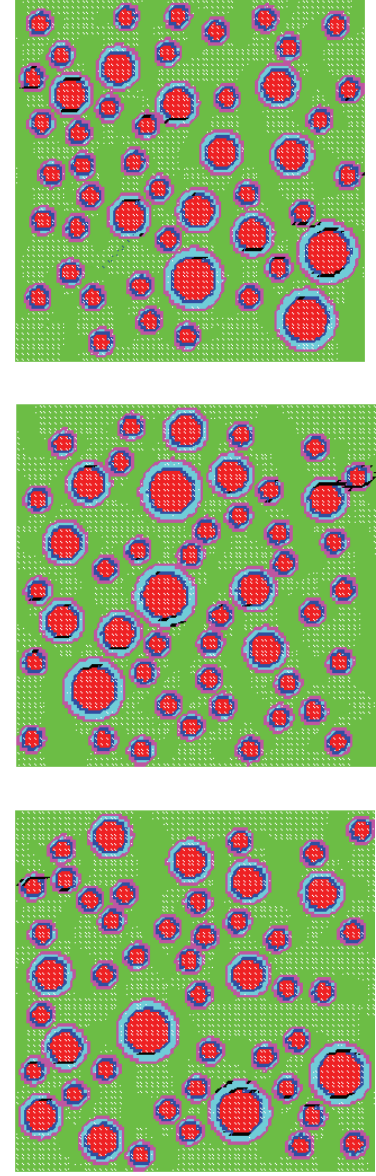
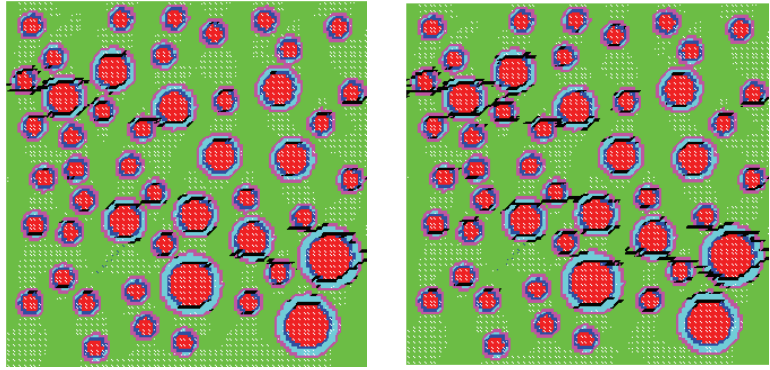

Specimen 1
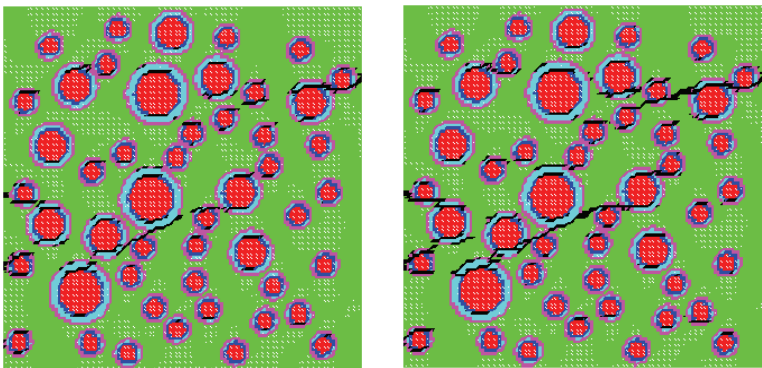

Specimen 2
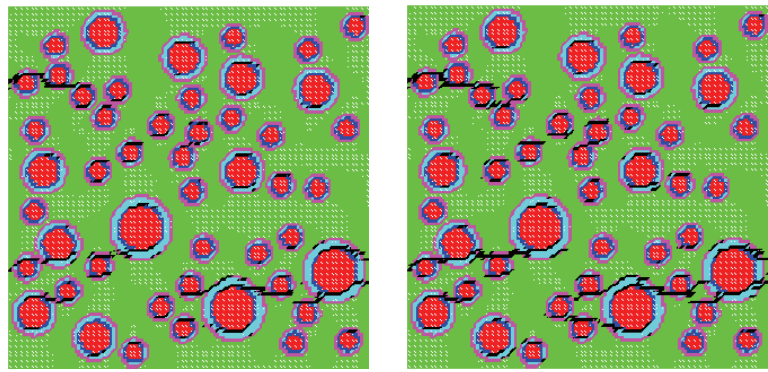

Specimen 3
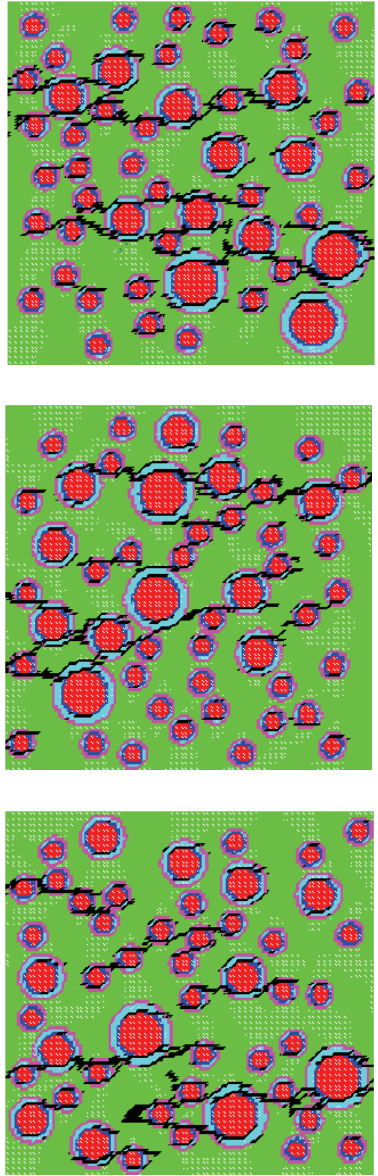

FIGURE 11: Propagation process of cracks of the RAC specimen by uniaxial tension.

\section{Conflict of Interests}

The authors declare that there is no conflict of interests.

\section{Acknowledgment}

This work is supported by the National Science Foundation of China, nos. 10972015, 11172015 and 91016026.

\section{References}

[1] F. H. Wittmann, P. E. Roelfstra, and H. Sadouki, "Simulation and analysis of composite structures," Materials Science and Engineering, vol. 68, no. 2, pp. 239-248, 1985.

[2] E. Schlangen and J. G. M. van Mier, "Simple lattice model for numerical simulation of fracture of concrete materials and structures," Materials and Structures, vol. 25, no. 9, pp. 534-542, 1992.

[3] E. Schlangen and E. J. Garboczi, "Fracture simulations of concrete using lattice models: computational aspects," Engineering Fracture Mechanics, vol. 57, no. 2-3, pp. 319-332, 1997.

[4] G. T. Liu and Z. M. Wang, "Numerical simulation of fracture of concrete materials using random aggregate model," Journal of Tsinghua University, vol. 36, no. 1, pp. 84-89, 1996 (Chinese).
[5] Y. J. Peng, B. K. Li, and B. Liu, "Numerical simulation of mesolevel mechanical properties of roller compacted concrete," Journal of Hydraulic Engineering, vol. 32, no. 6, pp. 19-22, 2001 (Chinese).

[6] X. L. Du, R. J. Tian, Y.-J. Peng, and Y. D. Tian, "Numerical simulation on the three-graded concrete beam under dynamic loading," World Information on Earthquake Engineering, vol. 24, no. 1, pp. 1-5, 2008 (Chinese).

[7] J. Z. Xiao, W. G. Li, Y. H. Fan, and X. Huang, "An overview of study on recycled aggregate concrete in China (1996-2011)," Construction and Building Materials, vol. 31, pp. 364-383, 2012.

[8] J. Z. Xiao, J. T. Du, and Q. Liu, "Numerical simulation on stressstrain curve of recycled concrete under uniaxial compression with lattice model," Journal of Building Materials, vol. 12, no. 5, pp. 511-514, 2009 (Chinese).

[9] Y. J. Peng, N. N. Dang, and J. Cheng, "A method on mesomechanics analysis for recycled aggregate concrete based on random aggregate model," in Proceedings of the Chinese Congress of Theoretical and Applied Mechanics (CCTAM '11), pp. 1-6, 2011, (Chinese).

[10] H. P. Zhou, Y. J. Peng, N. N. Dang, and J. W. Pu, "Numerical simulation of uniaxial compression performance for recycled concrete using micromechanics," Applied Mechanics and Materials, vol. 253, no. 1, pp. 550-554, 2013. 
[11] Y. J. Peng and M. Jin, "New complementary finite-element method based on base forces," Chinese Journal of Applied Mechanics, vol. 23, no. 4, pp. 649-652, 2006 (Chinese).

[12] Y. J. Peng and M. Jin, "Application of the base forces concept in finite element method on potential energy principle," Journal of Beijing Jiaotong University, vol. 31, no. 4, pp. 1-4, 2007 (Chinese).

[13] Y. J. Peng and M. Jin, "A new finite element method on potential energy principle by base forces," Journal of Beijing University of Technology, vol. 33, no. 7, pp. 687-692, 2007 (Chinese).

[14] Y. J. Peng and M. Jin, "Finite element method for arbitrary meshes based on complementary energy principle using base forces," Engineering Mechanics, vol. 24, no. 10, pp. 41-45, 2007 (Chinese).

[15] Y. J. Peng and Y. H. Liu, "Base force element method of complementary energy principle for large rotation problems," Acta Mechanica Sinica, vol. 25, no. 4, pp. 507-515, 2009.

[16] Y. J. Peng, Z. L. Dong, B. Peng, and Y. H. Liu, "Base force element method (BFEM) on potential energy principle for elasticity problems," International Journal of Mechanics and Materials in Design, vol. 7, no. 3, pp. 245-251, 2011.

[17] Y. J. Peng, Z. L. Dong, B. Peng, and N. N. Zong, "The application of $2 \mathrm{D}$ base force element method (BFEM) to geometrically nonlinear analysis," International Journal of Non-Linear Mechanics, vol. 47, no. 3, pp. 153-161, 2012.

[18] Y. C. Gao, "A new description of the stress state at a point with applications," Archive of Applied Mechanics, vol. 73, no. 3-4, pp. 171-183, 2003.

[19] J. C. Walraven and H. W. Reinhardt, "Theory and experiments on the mechanical behaviour of cracks in plain and reinforced concrete subjected to shear loading," Heron, vol. 26, no. 1, pp. $1-68,1981$.

[20] J. Z. Xiao, "Experimental investigation on complete stress-strain curve of recycled concrete under uniaxial loading," Journal of Tongji University, vol. 35, no. 11, pp. 1445-1449, 2007 (Chinese).

[21] J.-Z. Xiao and Y. Lan, "Investigation on the tensile behavior of recycled aggregate concrete," Journal of Building Materials, vol. 9, no. 2, pp. 154-158, 2006 (Chinese). 


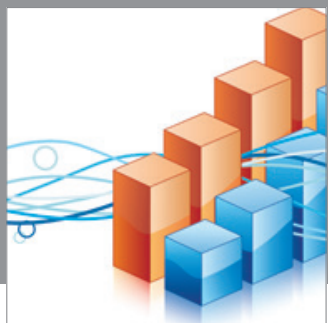

Advances in

Operations Research

mansans

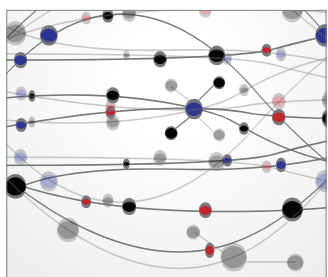

The Scientific World Journal
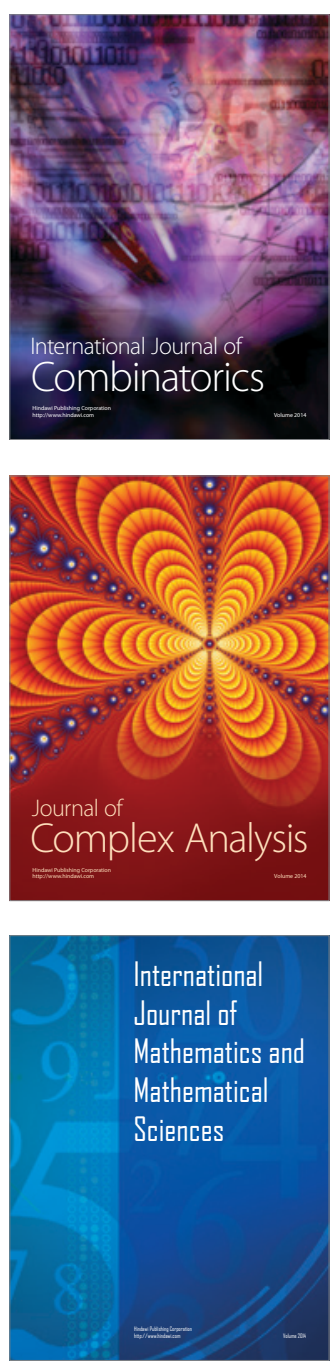
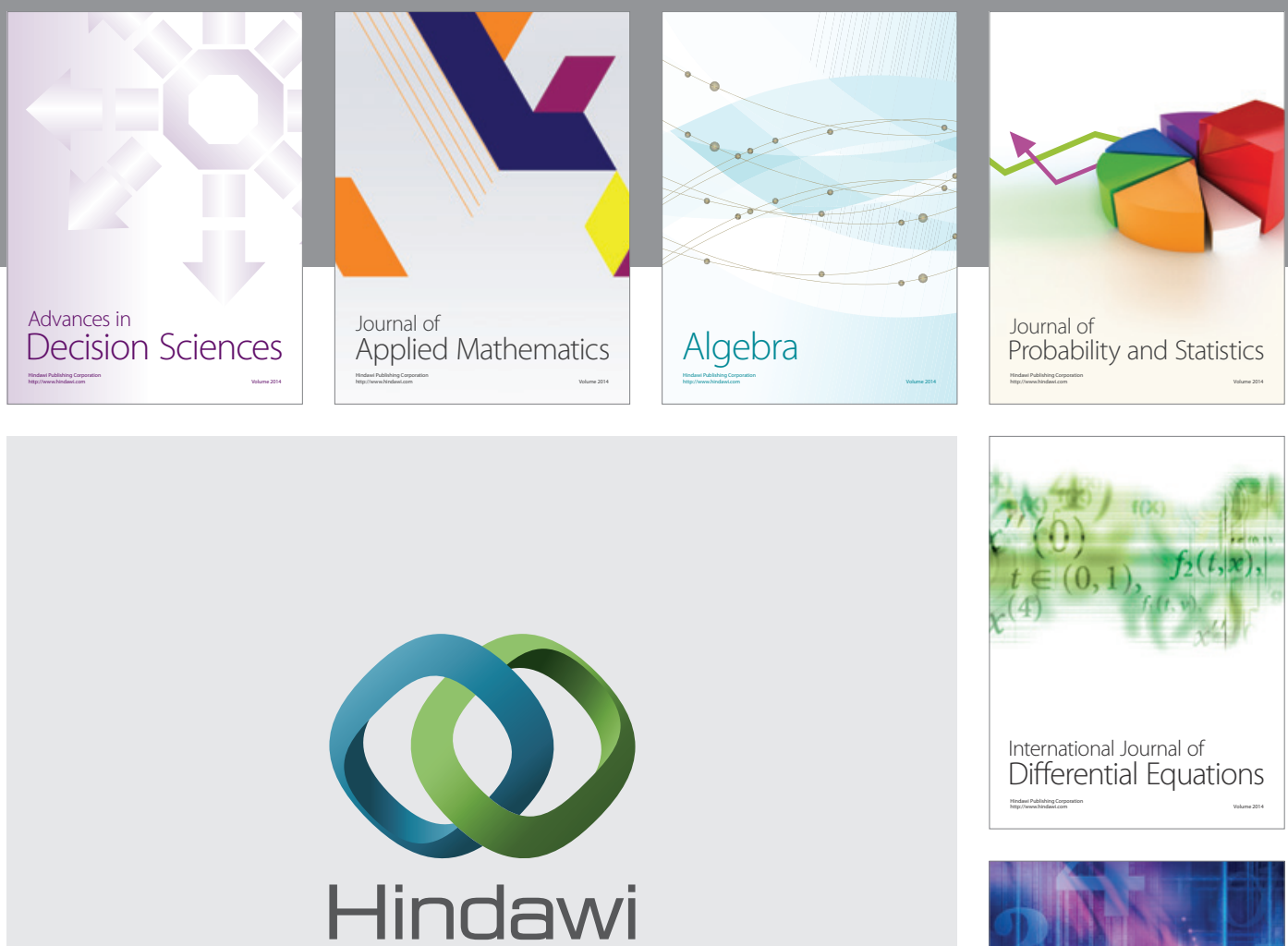

Submit your manuscripts at http://www.hindawi.com
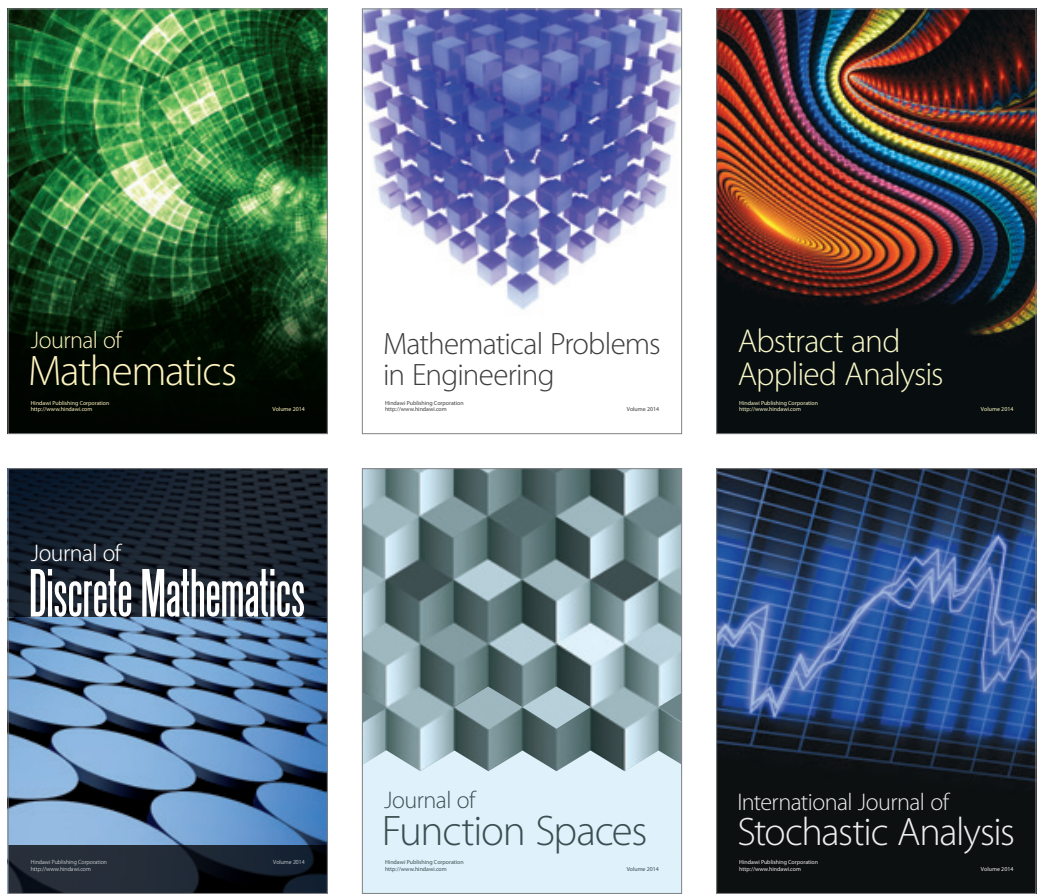

Journal of

Function Spaces

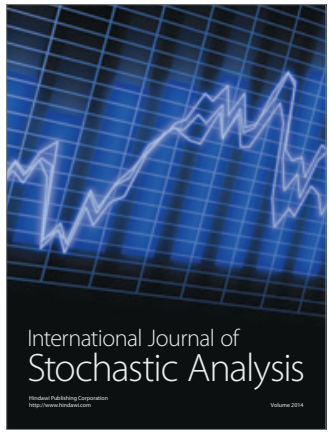

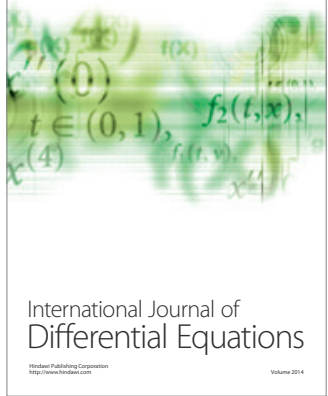
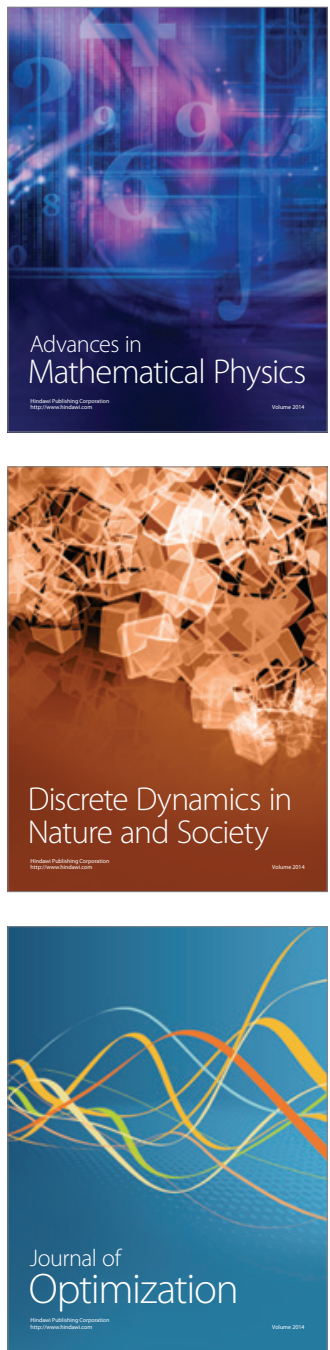